\title{
Novel Flexible Artificial Magnetic Conductor
}

\author{
M. E. de Cos and F. Las-Heras \\ Area de Teoría de la Señal y Comunicaciones, Departamento de Ingeniería Eléctrica, Universidad de Oviedo, Edificio Polivalente, \\ Modulo 8, Campus Universitario de Gijón, Asturias, 33203 Gijón, Spain
}

Correspondence should be addressed to M. E. de Cos, medecos@tsc.uniovi.es

Received 15 February 2012; Revised 29 April 2012; Accepted 30 April 2012

Academic Editor: Carles Fernández-Prades

Copyright (C) 2012 M. E. de Cos and F. Las-Heras. This is an open access article distributed under the Creative Commons Attribution License, which permits unrestricted use, distribution, and reproduction in any medium, provided the original work is properly cited.

\begin{abstract}
A novel flexible uniplanar AMC design is presented. An AMC prototype is manufactured using laser micromachining and it is characterized under flat and bent conditions by measuring its reflection coefficient phase in an anechoic chamber. The designed prototype shows broad AMC operation bandwidth (6.96\% and higher) and polarization angle independency. Its angular stability margin, when operating under oblique incidence, is also tested obtaining $\pm 8^{\circ}$ as limit for a $14.4 \mathrm{~cm} \times 14.4 \mathrm{~cm}$ prototype.
\end{abstract}

\section{Introduction}

Metamaterials have attracted a lot of attention in the recent years due to their unique properties in controlling the propagation of electromagnetic waves, which makes them able to solve some antennas and microwave circuits' problems. Between the metamaterial structures Artificial Magnetic Conductor (AMC) structures [1-10] are especially interesting in the design of highly efficient and low-profile antennas [11-17], due to their inherent in-phase reflection properties which in addition reduces the radiation to the body [18] in wearable applications [19-23].

Perfect Magnetic Conductors (PMCs) do not exist in nature. AMCs are synthesized PMCs and are dual to a Perfect Electric Conductor (PEC) from an electromagnetic point of view. AMCs exhibit a reflection coefficient with a magnitude value of 1 and a phase value of $0^{\circ}$ in the ideal lossless case. It is considered [1-3] that AMCs behave as PMCs over a certain frequency band, the so-called bandwidth of AMC performance or AMC operation bandwidth, which is generally defined in the range from $+90^{\circ}$ to $-90^{\circ}$, since in this range, the phase values would not cause destructive interference between direct and reflected waves.

AMCs are often implemented using two-dimensional periodic metallic lattices patterned on a conductor-backed dielectric surface. The research efforts recently focus on the development of low-cost AMCs that can be easily integrated in microwave and millimeter wave circuits. This requires the use of geometries without via holes [2, 4-9] (in contrast to designs consisting in patches with via holes [1]) as well as the use of a unilayer periodic Frequency Selective Surface (FSS) over a metallic ground plane (in contrast to multilayered FSSs [10]). In the unilayer case, the main drawback is the very narrow AMC operation bandwidth. By optimizing the unit cell geometry design and using a low relative dielectric permittivity substrate, which in addition reduces the cost, this can be overcome to some extent.

The interest in flexible AMCs is growing since it would be desirable to have AMC being object-shape-adapted for many applications as Radio Frequency Identification (RFID) tags over metallic objects [24], wearable antennas [19-23], and RCS reduction [25]. This would require the AMC to be flexible (especially in the case of objects with curved surfaces) but without losing its functionality.

In this paper, a novel compact uniplanar AMC design without via holes is presented and manufactured using a bendable dielectric substrate, which adds the advantage of flexibility to the resulting AMC structure, preserving its remarked features. In addition, the novel AMC exhibits other advantages as broad AMC operation bandwidth and high angular stability. 


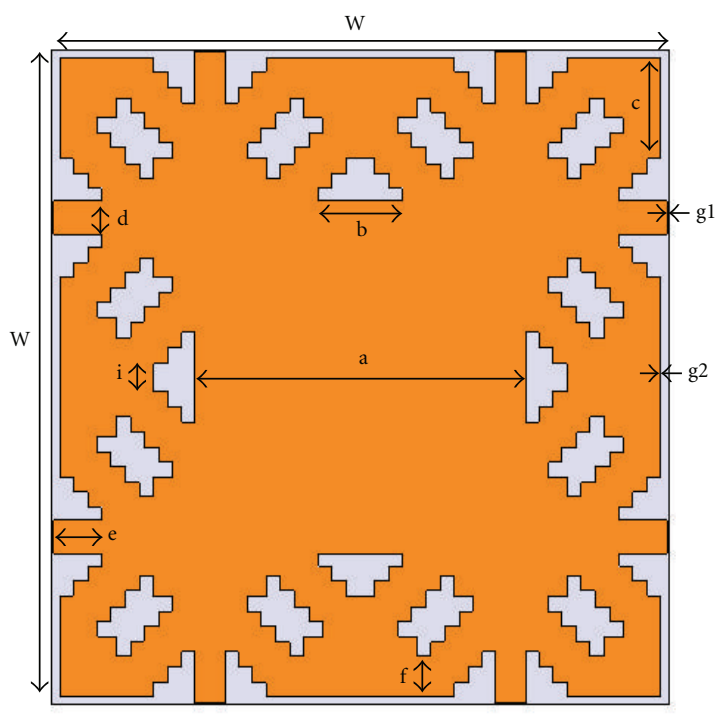

(a)

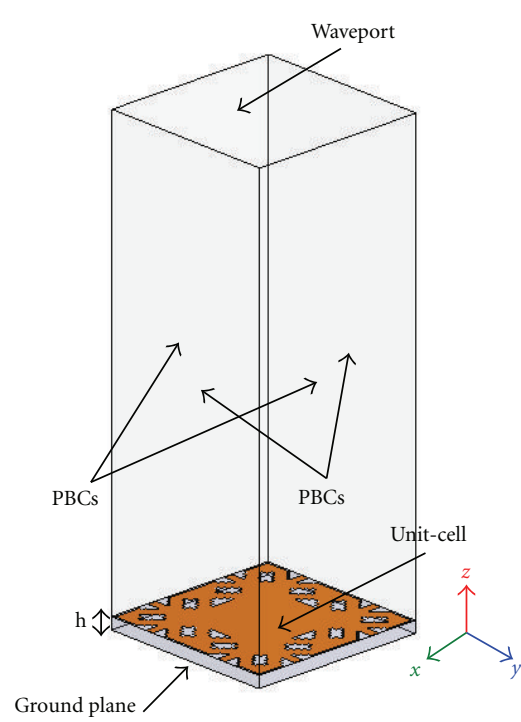

(b)

Figure 1: AMC unit-cell design: (a) unit-cell geometry top view and (b) simulation setup.

\section{AMC Desing}

In an AMC structure each unit cell implements a distributed parallel $L C$ network having one or more resonant frequencies. The resonance frequency is where the high impedance and AMC conditions occur and for a parallel $L C$ circuit is equal to $1 /(2 \pi \sqrt{L C})$, while in-phase reflection bandwidth is proportional to $\sqrt{L / C}$. In order to achieve an AMC structure at a certain frequency and with the desired bandwidth a designer can modify the unit cell geometry together with substrate's relative dielectric permittivity and thickness. The thicker the dielectric substrate is, the wider the AMC bandwidth is. The higher the dielectric substrate relative permittivity, the lower the resonance frequency but also, the lower the AMC bandwidth [6]. Finally, by changing the unit cell geometry the equivalent $L C$ resonant circuit is modified. In order to obtain a wider AMC operation bandwidth, it is necessary to increase $L$ and reduce $C$. $L$ can be increased using a thicker dielectric substrate and also including in the geometry narrow and long strips (lines). $C$ can be reduced by reducing substrate's relative dielectric permittivity $\varepsilon_{r}$ and increasing the gap between the metallization edge and the unit-cell edge (and so the gap between adjacent unit cells). In order to obtain both compact size and broad AMC operation bandwidth, a trade-off solution regarding $\varepsilon_{r}$ and substrate thickness has to be adopted.

Taking all these considerations into account, a novel uniplanar AMC at $6 \mathrm{GHz}$ has been designed using ROGER3003 substrate with a thickness $h=0.762 \mathrm{~mm}$, relative dielectric permittivity $\varepsilon_{r}=3.0$, and loss tangent $t g_{\delta}=0.0013$.

Finite Element Method (FEM) together with the BlochFloquet theory is used in simulation to search for the frequency band in which the periodic structure behaves as an AMC. The AMC reflection coefficient for a uniform incident plane wave is simulated using HFSS of Ansoft, modeling a single cell of the structure with periodic boundary conditions (PBC) on its sides and resembling the modeling of an infinite structure $[1,6,7,9]$. The periodic structure is illuminated, launching normal plane waves using a waveport positioned a half-wavelength above it (see Figure 1(b)). The phase reference plane is taken on the periodic surface. The phase of the reflection coefficient of the AMC plane is compared to that of a PEC plane taken as reference and placed in identical position, in the same way as in [1].

The geometry of the AMC unit cell is shown in Figure 1(a). Its design has taken into account that the AMC will be subsequently bent. When defining " $h$," the thickness of the substrate is taken into account that it is desired to be as thin as possible considering the thickness commercially available and to be bendable. Also the thinner the dielectric substrate, the higher the AMC's angular stability under oblique incidence. Very narrow strips have been avoided since they may break when the AMC is bent, despite the long and narrow strips increasing the bandwidth of operation as AMC. Internal gaps in the geometry are used to modify the equivalent $C$ and thus adjust the frequency and bandwidth, as desired (in addition to what has been explained earlier in this section). The final optimized dimensions for operation at $6 \mathrm{GHz}$ are detailed in Table 1. From the simulated reflection coefficient phase of the designed AMC structure (see Figure 2), it can be concluded that the resonance frequency is $6 \mathrm{GHz}$ with an AMC operation bandwidth of $500 \mathrm{MHz}$ (8.33\% with respect to the central frequency) which is a broad bandwidth for a low-profile AMC $\left(\lambda_{0} / 65.6\right.$ at $6 \mathrm{GHz}$ ).

The AMC performance under different polarization of the electrical incident field (under normal incidence) and under oblique incidence is very important in some of the AMC intended applications as for example RFID tags, or wearable antennas. In the case of RFID tags when combining 


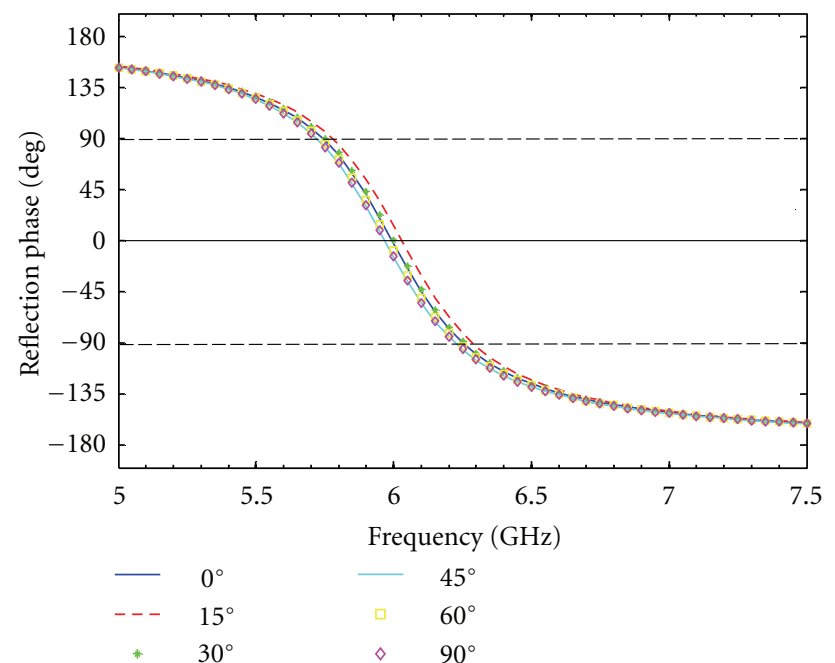

FIGURE 2: Simulated reflection phase of the AMC surface for different incident field $\left(\mathbf{E}_{\text {inc }}\right)$ polarization angles $=0^{\circ}, 15^{\circ}, 30^{\circ}, 45^{\circ}$, $60^{\circ}$, and $90^{\circ}$.

TABLE 1: Unit-cell dimensions.

\begin{tabular}{lccccccccc}
\hline \multicolumn{10}{c}{ Dimensions $(\mathrm{mm})$} \\
\hline$W$ & $a$ & $b$ & $c$ & $d$ & $e$ & $f$ & $g 1$ & $g 2$ & $i$ \\
12.0 & 6.456 & 1.646 & 1.827 & 0.609 & 0.945 & 0.730 & 0.032 & 0.154 & 0.522 \\
\hline
\end{tabular}

the AMC with the antenna, the angular stability of the AMC will influence the antenna radiation performance, and this will have direct impact on the angular reading range of the final RFID tag depending on the position of the reader with respect to the tagged object. Following this, an AMC design with as higher angular stability as possible is desirable.

Aiming to study the angular stability margin [26] of the presented structure, the reflection coefficient phase versus frequency for different incident angles $\theta_{\text {inc }}$ between $0^{\circ}$ and $60^{\circ}$ has been simulated for transverse-electric- (TE-) polarized waves. The absolute and relative deviations of the resonant frequency which are, respectively, $50 \mathrm{MHz}, 0.82 \%$ for $\theta_{\text {inc }}=40^{\circ}$ and $100 \mathrm{MHz}, 1.6 \%$ for $\theta_{\text {inc }}=60^{\circ}$ can be obtained from Figure 3. The AMC operation bandwidth is slightly reduced from $\theta_{\text {inc }}=40^{\circ}$. From these obtained results, it can be concluded that the presented AMC design is highly stable as its angular margin ranges from $0^{\circ}$ to $60^{\circ}$ preserving an AMC operation bandwidth of $260 \mathrm{MHz}$ in which the operation frequency of $6 \mathrm{GHz}$ is always included.

\section{Flexible AMC's Characterization}

In order to validate the simulation results, a $12 \times 12$ cells planar AMC prototype has been manufactured using laser micromachining to be measured.

There are many arbitrary ways of bending a flexible AMC. Two typical different bending patterns have been selected and tested (see Figure 4): a "creeping" pattern (henceforth referred to as creeping prototype) which can be caused in textile AMC integrated in the garment, for example

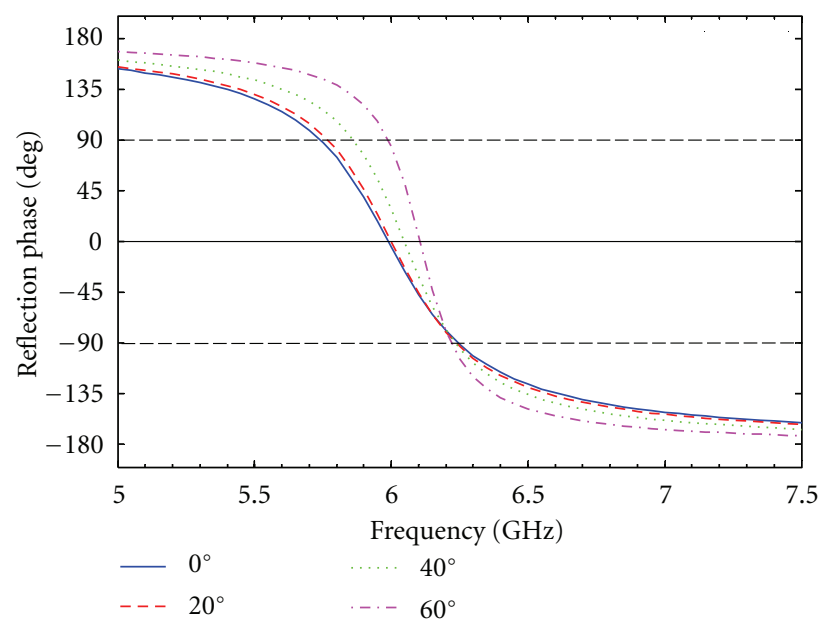

FIGURE 3: Simulated reflection phase of the AMC surface for TE polarizations for different incident angles $\theta_{\text {inc }}=0^{\circ}, 15^{\circ}, 30^{\circ}, 45^{\circ}$, and $60^{\circ}$.

when the arm is bent at the elbow, and a "smooth" pattern (henceforth referred as smooth prototype) which can be caused in the torso or in the shoulder.

3.1. Measurement Setup in Anechoic Chamber. Two-horn antenna probes working in the band $5-7 \mathrm{GHz}$ have been chosen as Tx and Rx (see Figure 5) in the measurement setup (similar to the one used in $[7,9]$ ), being $3 \mathrm{~m}$ the separation between each probe and the object under test. The measured prototypes physical size is $14.4 \mathrm{~cm} \times 14.4 \mathrm{~cm}$ (PEC and AMC have the same size). For the upper frequency ( $f=7 \mathrm{GHz})$, the far field distance $\left(\mathrm{RFF}=2 D^{2} / \lambda\right)$ is $\mathrm{RFF}_{7 \mathrm{GHz}} \sim 0.97 \mathrm{~m}$, whereas for the lower frequency $(f=5 \mathrm{GHz})$ it decreases until $\mathrm{RFF}_{, 5 \mathrm{GHz}} \sim 0.69 \mathrm{~m}$. Thus, the prototypes have been measured in far field conditions.

3.2. Reflection Phase Measurement for AMC Band Determination. To calculate the reflection coefficient of the AMC structure the same methodology as for the full-wave simulation, based on the utilization of a reference measurement (metallic plate) $[1,7]$, is followed.

Firstly the flat AMC has been characterized and then the prototype has been bent and measured.

The measured reflection phase of the flat and bent manufactured prototypes for normal incidence conditions is shown in Figure 6. The flat prototype has the resonance at $6.178 \mathrm{GHz}$ which means a $2.9 \%$ deviation with respect to the simulation $(6.0 \mathrm{GHz})$, very probably due to under-etching in the laser micromachining.

From Table 2 and Figure 6, it can be observed that almost there is no frequency shift for the manufactured creeping bent prototype with respect to the flat prototype resonance, whereas the smooth bent prototype has its resonance at $6.208 \mathrm{GHz}$, which means just a $1.69 \%$ deviation with respect to the flat prototype. The flat prototype shows a $430 \mathrm{MHz}$ (6.96\%) AMC operation bandwidth in good agreement with the simulated value $(8.33 \%)$ (see Figure 2), whereas the 

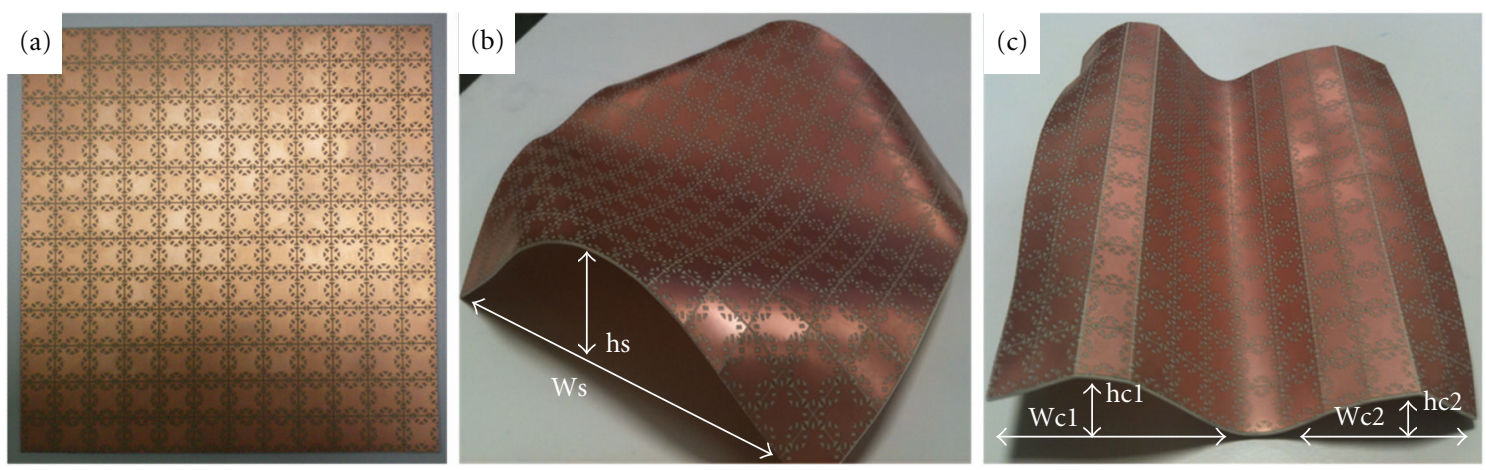

FIGURE 4: (a) Flat manufactured prototype and bending patters: (b) smooth prototype with Ws $=125 \mathrm{~mm}$ and hs $=30 \mathrm{~mm}$; (c) creeping prototype with $\mathrm{Wc1}=60 \mathrm{~mm}, \mathrm{Wc} 2=52 \mathrm{~mm}, \mathrm{hcl}=18 \mathrm{~mm}$, and hc2 $=15 \mathrm{~mm}$.

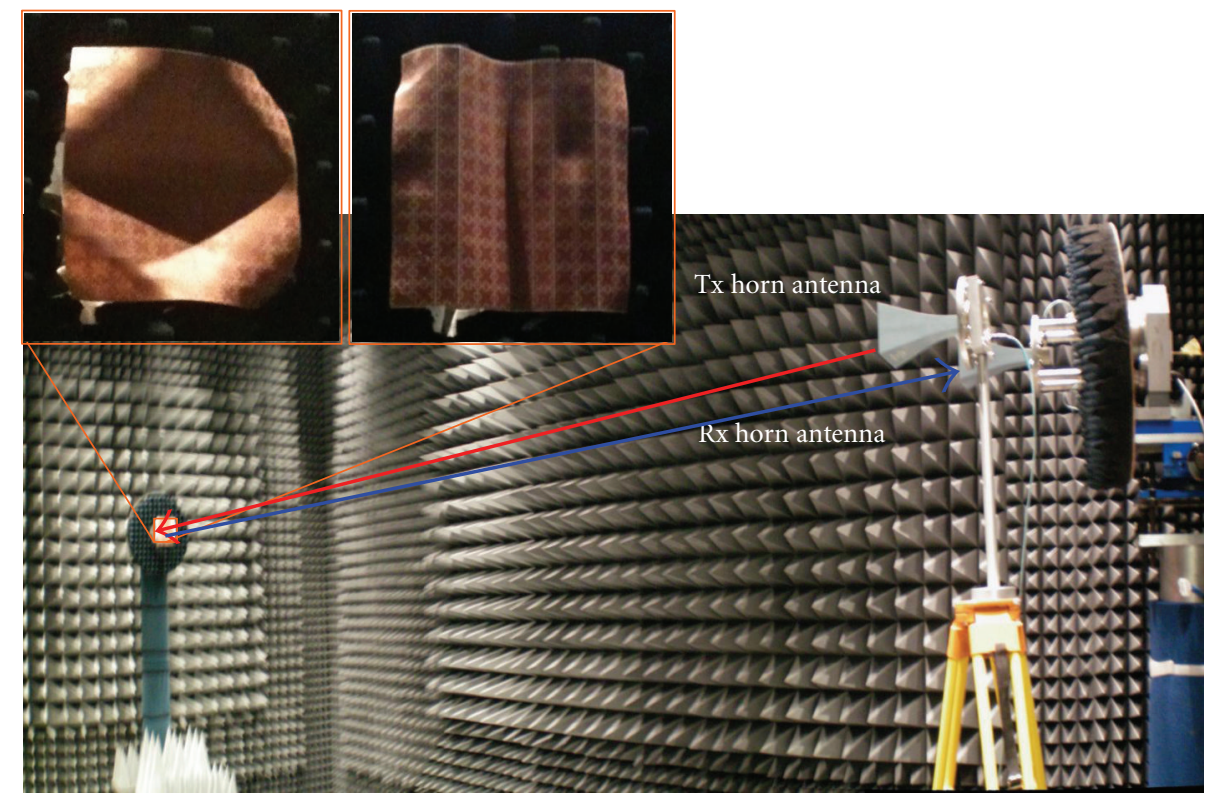

FIGURE 5: Reflection coefficient measurement setup in anechoic chamber.

TABle 2: Prototypes comparison.

\begin{tabular}{lccc}
\hline Prototype & $\begin{array}{c}\text { Resonance } \\
\text { frequency }(\mathrm{GHz})\end{array}$ & $\begin{array}{c}\text { Frequency deviation } \\
\text { with respect to flat } \\
(\%)\end{array}$ & $\begin{array}{c}\text { Measured } \\
\text { bandwidth } \\
(\%)\end{array}$ \\
\hline Flat & 6.178 & 0 & 6.96 \\
Smooth & 6.073 & 1.69 & 8.02 \\
Creeping & 6.208 & 0.48 & 10.07 \\
\hline
\end{tabular}

creeping bent prototype exhibits $625 \mathrm{MHz}(10.07 \%)$ and the smooth bent AMC shows $487 \mathrm{MHz}$ (8.02\%), even slightly wider than that of the flat prototype.

\subsection{Reflection Phase Characterization for Different Field Polar-} ization Angles. The reflection phase stability of the manufactured AMC prototypes with respect to the incident field polarization angle $(\varphi)$ has been also tested through measurements.

It has been proved that under normal incidence $\left(\theta_{\text {inc }}=\right.$ $0^{\circ}$ ) the flat prototype presents the same reflection phase for any polarization due to the unit cell symmetry (see Figure 7). In the case of the bent prototype, this invariance with respect to the polarization angle is also present.

3.4. Reflection Phase Characterization for Different Incidence Angles. The angular stability under oblique incidence has been analyzed for both the smooth and the creeping prototypes. The reflection coefficient phase versus frequency, for different incident angles $\theta_{\text {inc }}$, has been measured. For the flat prototype, resonance conditions are met within an angular margin of $\theta_{\text {inc }}= \pm 10^{\circ}$ (see Figure 8), whereas for the smooth and creeping bent prototypes the obtained angular margin is $\theta_{\text {inc }}= \pm 8^{\circ}$ (see Figures 9 and 10). The differences between simulation and measurements can be 


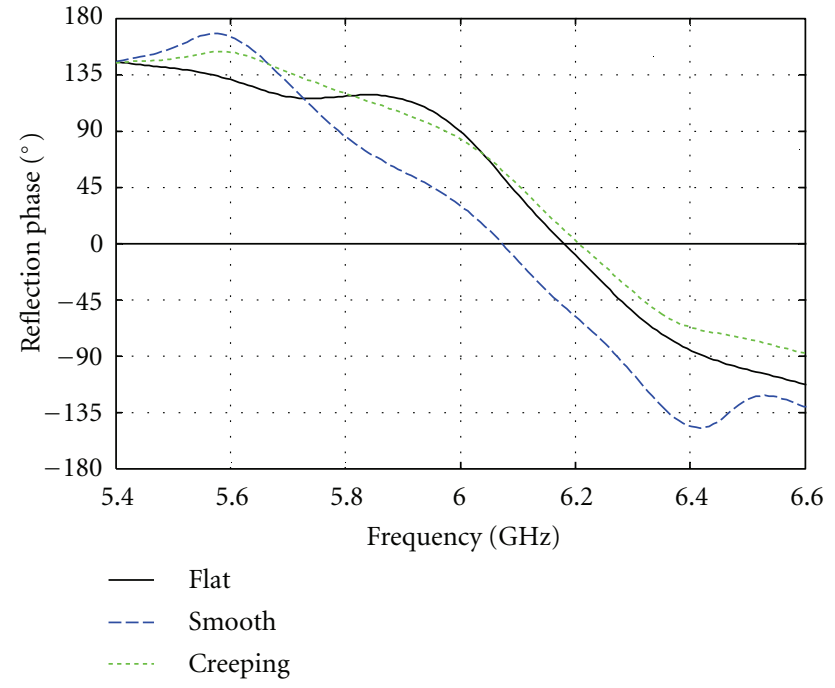

Figure 6: Measured reflection coefficient phase of the flat and bent prototypes versus frequency.

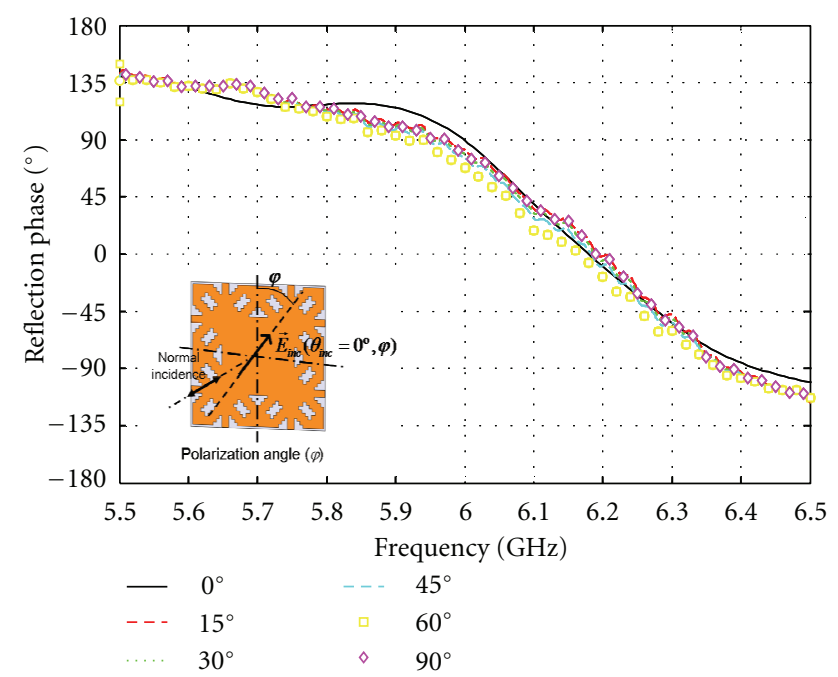

FIGURE 7: Reflection phase of the manufactured prototype for different incident field $\left(\mathbf{E}_{\text {inc }}\right)$ polarization angles $(\varphi)$.

attributed to the small and finite size of the manufactured prototype, which leads to some nulls in the scattered field amplitude, depending on the incident angle and the distance between the prototype and the antennas. This also happens when characterizing a finite-size PEC following the same procedure.

The presented results show that it is possible to obtain a flexible AMC without reducing the bandwidth of AMC performance with respect to a rigid AMC that uses the same unit-cell design and preserving its angular stability under oblique incidence.

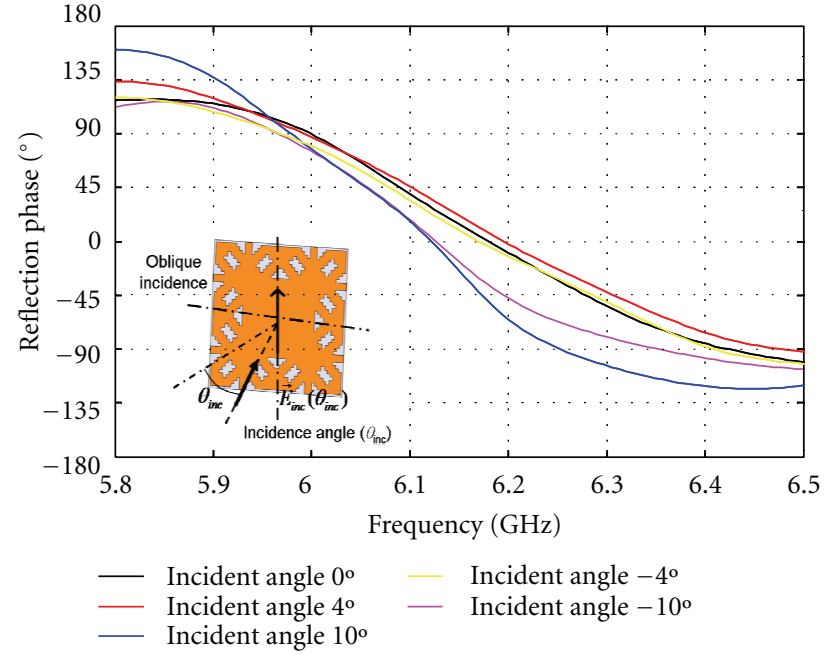

FIGURE 8: Reflection phase of the manufactured flat prototype for different incident angles $\left(\theta_{\text {inc }}\right)$.

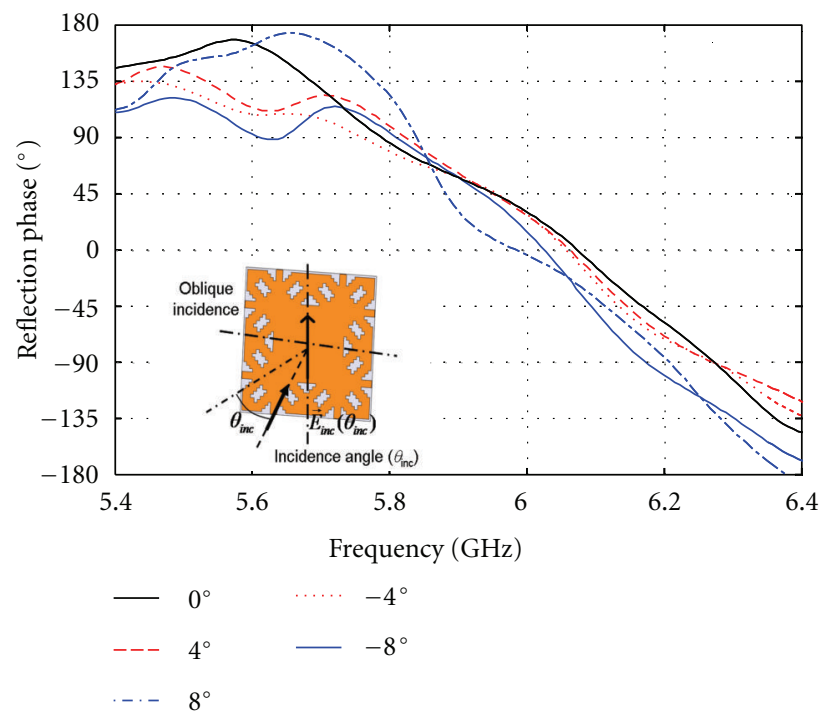

FIGURE 9: Reflection phase of the manufactured smooth prototype for different incident angles $\left(\theta_{\text {inc }}\right)$.

\section{Conclusions}

A novel flexible uniplanar AMC design based on FEM simulations has been presented. A prototype has been manufactured using a bendable dielectric substrate and it has been characterized by means of reflection coefficient phase measurements in anechoic chamber under flat and bent conditions. Broad AMC operation bandwidth, polarization angle independency under normal incidence, and high angular stability under oblique incidence have been found.

The presented uniplanar low-profile design without via holes, together with the flexible characteristic, low cost, simple fabrication, and integration, makes it very attractive 


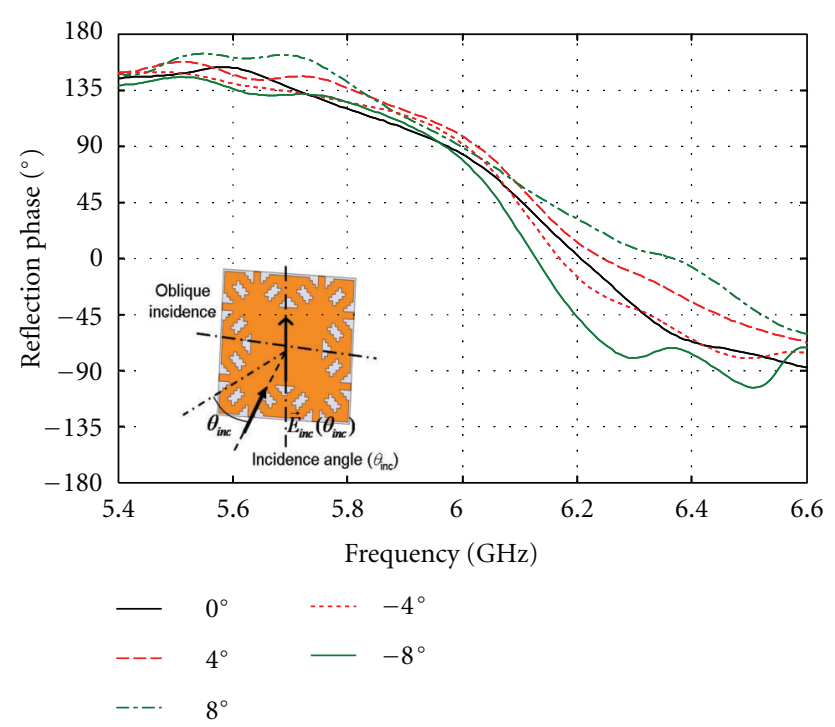

Figure 10: Reflection phase of the manufactured creeping prototype for different incident angles $\left(\theta_{\text {inc }}\right)$.

for applications involving antennas in RFID tags, wearable systems, and RCS reduction. Also it could be used as part of Microwave Integrated Circuits (MICs).

\section{Acknowledgments}

This work has been supported by the Ministerio de Ciencia e Innovación of Spain/FEDER under Projects TEC201124492 (iScat) and CONSOLIDER-INGENIO CSD200800068 (TERASENSE), by the Gobierno del Principado de Asturias (PCTI)/FEDER-FSE under Projects EQUIP08-06, FC09-COF09-12, EQUIP10-31, and PC10-06 (FLEXANT).

\section{References}

[1] D. Sievenpiper, L. Zhang, R. F. Jimenez Broas, N. G. Alexöpolous, and E. Yablonovitch, "High-impedance electromagnetic surfaces with a forbidden frequency band," IEEE Transactions on Microwave Theory and Techniques, vol. 47, no. 11, pp. 2059-2074, 1999.

[2] F. R. Yang, K. P. Ma, M. Yongxi Qian, and T. Itoh, "A uniplanar compact photonic-bandgap (UC-PBG) structure and its applications for microwave circuits," IEEE Transactions on Microwave Theory and Techniques, vol. 47, no. 8, pp. 15091514, 1999.

[3] F. Yang and Y. Rahmat-Samii, Electromagnetic Band-Gap Structures in Antenna Engineering, The Cambridge RF and Microwave Engineering Series, Cambridge University, 2008.

[4] J. McVay, N. Engheta, and A. Hoorfar, "High impedance metamaterials surfaces using Hilbert-curve inclusions," IEEE Microwave and Wireless Components Letters, vol. 14, no. 3, pp. 130-132, 2004.

[5] Y. Kim, F. Yang, and A. Z. Elsherbeni, "Compact artificial magnetic conductor designs using planar square spiral geometries," Progress in Electromagnetics Research, vol. 77, pp. 43-54, 2007.
[6] M. E. De Cos, F. L. Heras, and M. Franco, "Design of planar artificial magnetic conductor ground plane using frequencyselective surfaces for frequencies below $1 \mathrm{GHz}$," IEEE Antennas and Wireless Propagation Letters, vol. 8, pp. 951-954, 2009.

[7] M. E. De Cos, Y. Álvarez, and F. Las-Heras, "Planar artificial magnetic conductor: design and characterization setup in the RFID SHF band," Journal of Electromagnetic Waves and Applications, vol. 23, no. 11-12, pp. 1467-1478, 2009.

[8] D. J. Kern, D. H. Werner, A. Monorchio, L. Lanuzza, and M. J. Wilhelm, "The design synthesis of multiband artificial magnetic conductors using high impedance frequency selective surfaces," IEEE Transactions on Antennas and Propagation, vol. 53, no. 1 I, pp. 8-17, 2005.

[9] M. E. De Cos, Y. Álvarez, R. C. Hadarig, and F. Las-Heras, "Novel SHF-band uniplanar artificial magnetic conductor," IEEE Antennas and Wireless Propagation Letters, vol. 9, pp. 4447, 2010.

[10] A. Monorchio, G. Manara, and L. Lanuzza, "Synthesis of artificial magnetic conductors by using multilayered frequency selective surfaces," IEEE Antennas and Wireless Propagation Letters, vol. 1, pp. 196-199, 2002.

[11] F. Yang and Y. Rahmat-Samii, "Reflection phase characterizations of the EBG ground plane for low profile wire antenna applications," IEEE Transactions on Antennas and Propagation, vol. 51, no. $10 \mathrm{I}$, pp. 2691-2703, 2003.

[12] J. McVay, A. Hoorfar, and N. Engheta, "Small dipole antenna near peano high-impedance surfaces," in Proceedings of the IEEE Antennas and Propagation Society Symposium, vol. 1, pp. 305-308, June 2004.

[13] H. Mosallaei and K. Sarabandi, "Antenna miniaturization and bandwidth enhancement using a reactive impedance substrate," IEEE Transactions on Antennas and Propagation, vol. 52, no. 9, pp. 2403-2414, 2004.

[14] L. Akhoondzadeh-Asl, D. J. Kern, P. S. Hall, and D. H. Werner, "Wideband dipoles on electromagnetic bandgap ground planes," IEEE Transactions on Antennas and Propagation, vol. 55, no. 9, pp. 2426-2434, 2007.

[15] J. Liang and H. Y. D. Yang, "Radiation characteristics of a microstrip patch over an electromagnetic bandgap surface," IEEE Transactions on Antennas and Propagation, vol. 55, no. 6, pp. 1691-1697, 2007.

[16] A. P. Feresidis, G. Goussetis, S. Wang, and J. C. Vardaxoglou, "Artificial magnetic conductor surfaces and their application to low-profile high-gain planar antennas," IEEE Transactions on Antennas and Propagation, vol. 53, no. 1 I, pp. 209-215, 2005.

[17] J. R. Sohn, K. Y. Kim, H. S. Tae, and J. H. Lee, "Comparative study on various artificial magnetic conductors for low-profile antenna," Progress in Electromagnetics Research, vol. 61, pp. 2737, 2006.

[18] E. Rajo-Iglesias, L. Inclán-Sánchez, and Q. Quevedo-Teruel, "Back radiation reduction in patch antennas using planar soft surfaces," Progress In Electromagnetics Research Letters, vol. 6, pp. 123-130, 2009.

[19] S. Zhu and R. Langley, "Dual-band wearable textile antenna on an EBG substrate," IEEE Transactions on Antennas and Propagation, vol. 57, no. 4, pp. 926-935, 2009.

[20] M. Mantash, A-C. Tarot, S. Collardey, and K. Mabjoubi, "Dual-band antenna for W-LAN applications with EBG," in Proceedings of the 5th International Congress on Advanced Electromagnetic Materials in Microwave and Optics (Metamaterials '11), pp. 456-458, Barcelona, Spain, October 2011.

[21] M. Mantash, A. C. Tarot, S. Collardey, and K. Mahdjoubi, "Dual-band CPW-fed G-antenna using an EBG structure," in 
Proceedings of the 6th Loughborough Antennas and Propagation Conference (LAPC'10), pp. 453-456, November 2010.

[22] P. Salonen and Y. Rahmat-Samii, "Textile antennas: effects of antenna bending on input matching and impedance bandwidth," IEEE Aerospace and Electronic Systems Magazine, vol. 22, no. 3, pp. 10-14, 2007.

[23] P. Salonen, F. Yang, Y. Rahmat-Samii, and M. Kivikoski, "WEBGA-Wearable electromagnetic band-gap antenna," in Proceedings of the IEEE Antennas and Propagation Society Symposium, vol. 1, pp. 451-454, Monterrey, Calif, USA, June 2004.

[24] R. C. Hadarig, M. E. De Cos Gomez, Y. Álvarez, and F. LasHeras, "Novel bow-tie-AMC combination for 5.8-GHz RFID tags usable with metallic objects," IEEE Antennas and Wireless Propagation Letters, vol. 9, pp. 1217-1220, 2010.

[25] M. E. de Cos, Y. Álvarez, and F. Las-Heras, "A novel approach for RCS reduction using a combination of artificial magnetic conductors," Progress in Electromagnetics Research, vol. 107, pp. 147-159, 2010.

[26] C. R. Simovski, P. De Maagt, S. A. Tretyakov, M. Paquay, and A. A. Sochava, "Angular stabilisation of resonant frequency of artificial magnetic conductors for TE-incidence," Electronics Letters, vol. 40, no. 2, pp. 92-93, 2004. 

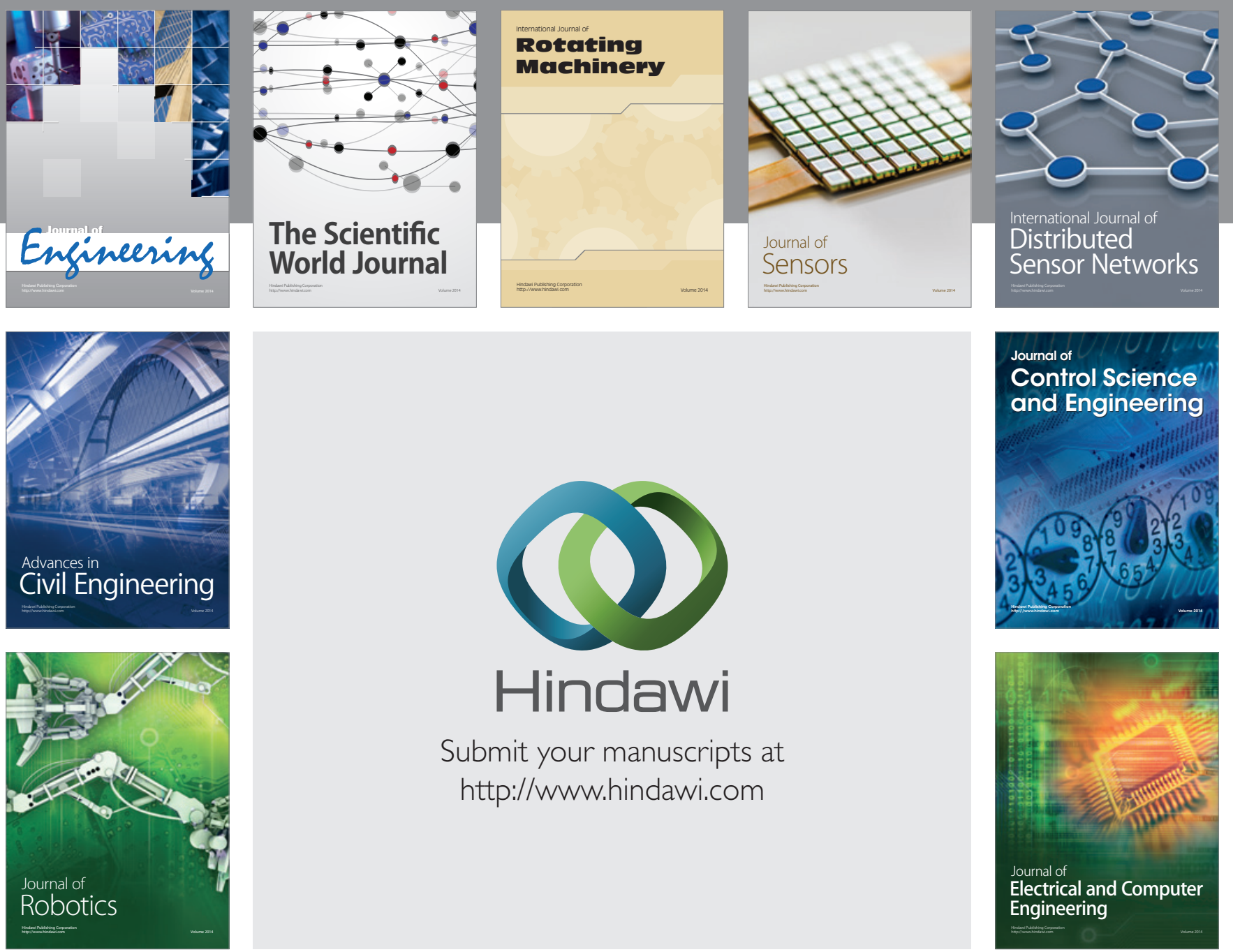

Submit your manuscripts at

http://www.hindawi.com
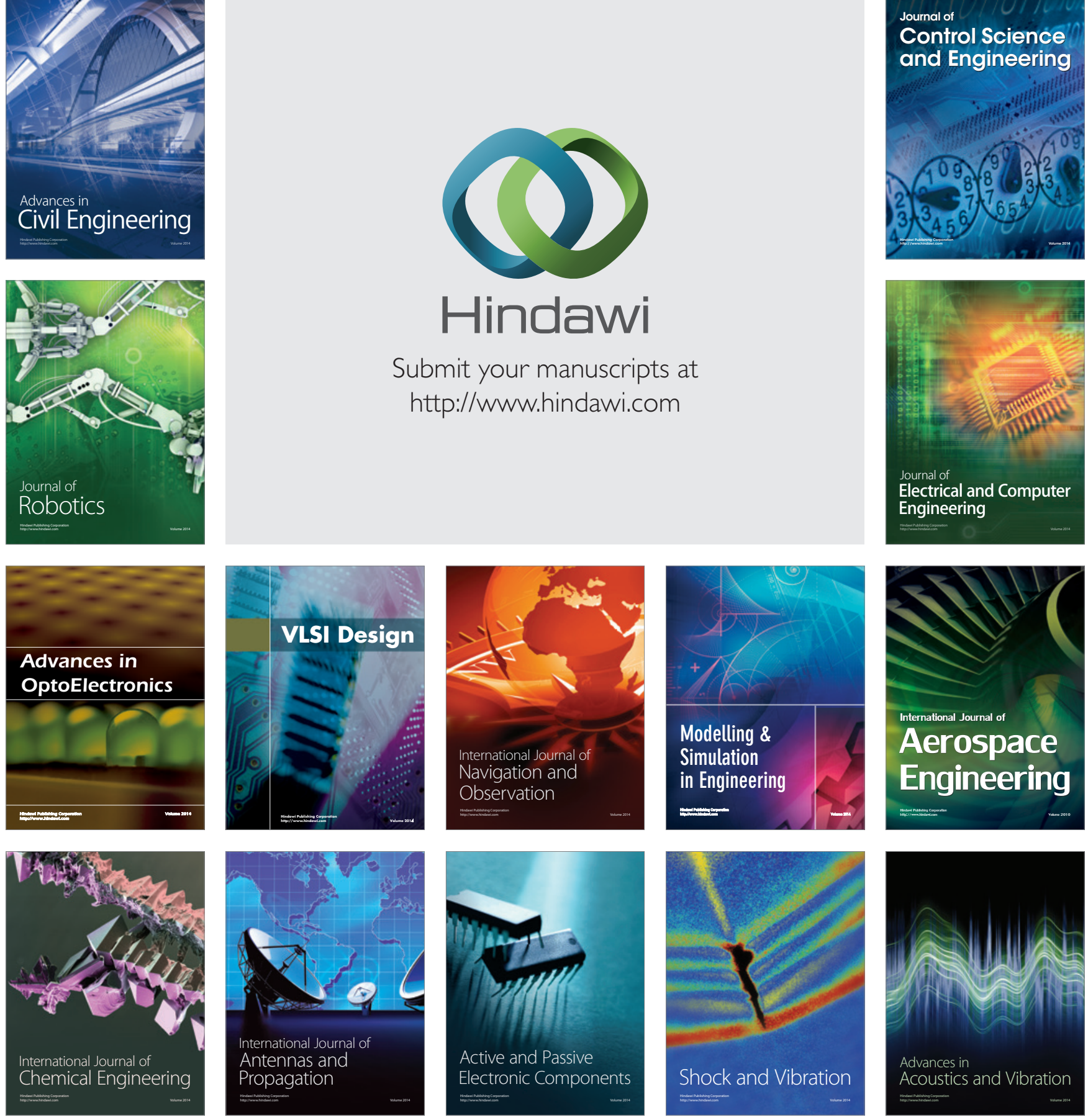